\title{
Sample pooling is a viable strategy for SARS-CoV-2 detection in low-prevalence settings
}

\author{
Brian S. W. Chong ${ }^{1}$, Thomas Tran ${ }^{1}$, Julian Druce ${ }^{1}$, Susan A. Ballard ${ }^{2}$, \\ Julie A. Simpson ${ }^{3}$, Mike Catton ${ }^{1}$ \\ ${ }^{1}$ Victorian Infectious Diseases Reference Laboratory, Melbourne Health at the Peter Doherty \\ Institute for Infection and Immunity, Melbourne, Vic, Australia; ${ }^{2}$ Microbiological Diagnostic \\ Unit Public Health Laboratory, The University of Melbourne at the Peter Doherty Institute for \\ Infection and Immunity, Melbourne, Vic, Australia; ${ }^{3}$ Centre for Epidemiology and Biosta- \\ tistics, Melbourne School of Population and Global Health, The University of Melbourne, \\ Melbourne, Vic, Australia
}

\section{Summary}

The severe acute respiratory syndrome coronavirus 2 (SARS-CoV-2) pandemic has significantly increased demand on laboratory throughput and reagents for nucleic acid extraction and polymerase chain reaction (PCR). Reagent shortages may limit the expansion of testing required to scale back containment measures. The aims of this study were to investigate the viability of sample pooling as a strategy for increasing test throughput and conserving PCR reagents; and to report our early experience with pooling of clinical samples.

A pre-implementation study was performed to assess the sensitivity and theoretical efficiency of two, four, and eightsample pools in a real-time reverse transcription PCRbased workflow. A standard operating procedure was developed and implemented in two laboratories during periods of peak demand, inclusive of over 29,000 clinical samples processed in our laboratory.

Sensitivity decreased (mean absolute increase in cycle threshold value of $0.6,2.3$, and 3.0 for pools of two, four, and eight samples, respectively) and efficiency increased as pool size increased. Gains from pooling diminished at high disease prevalence. Our standard operating procedure was successfully implemented across two laboratories. Increased workflow complexity imparts a higher risk of errors, and requires risk mitigation strategies. Turnaround time for individual samples increased, hence urgent samples should not be pooled.

Pooling is a viable strategy for high-throughput testing of SARS-CoV-2 in low-prevalence settings.

Key words: SARS-CoV-2; COVID-19; pooling; polymerase chain reaction.

Received 25 August, revised 17 September, accepted 18 September 2020 Available online 22 September 2020

\section{INTRODUCTION}

Timely, scalable, and accurate diagnostic testing for SARSCoV-2 underpins the public health response to the COVID19 pandemic and clinical care of suspected cases. Real-time reverse transcription-polymerase chain reaction (rRT-PCR) on a respiratory sample-most commonly an upper respiratory tract swab-is the main diagnostic modality for detection of SARS-CoV-2. ${ }^{1}$ However, global shortages of PCR reagents and consumables have constrained testing capacity, and may continue to limit the expansion of testing that countries will need to safely scale back containment measures. Group testing, or pooling of patient samples has previously been employed in mass screening, both for nucleic acid testing and immunoassays. ${ }^{2,3}$ This approach may increase the throughput of PCR and improve the utilisation of PCR reagents during difficult times for routine RT-PCRbased diagnostic workflow. A recent Californian study has described the process of pooling for SARS-CoV-2 testing on a relatively small number of samples, with two samples positive out of 2888 tested. ${ }^{4}$ In this study, we explore the relative sensitivity of sample pools of varying sizes compared to standard single-specimen SARS-CoV-2 rRT-PCR protocols, the relative efficiency gains yielded by different pool sizes, and the effect of disease prevalence on these gains. We have also developed a standard operating procedure and tested its implementation, both in our own busy diagnostic service and in another laboratory. Finally, we summarise our experience with processing over 29,000 diagnostic samples in pools of two different sizes during two periods of peak diagnostic demand.

\section{METHODS}

\section{Study setting}

The Victorian Infectious Diseases Reference Laboratory (VIDRL) is a reference laboratory located in Melbourne, Australia. VIDRL performed all of the SARS-CoV-2 diagnostic testing for the state of Victoria in the early days of the pandemic. In anticipation of increasing demand for testing, a preimplementation study for sample pooling was performed with defined samples. Pooling of clinical specimens was formally performed during two periods of high demand in March and May 2020.

\section{Pre-implementation study}

Pool sizes consisting of two, four, and eight pre-defined samples were used for this study. Each pool contained a single known SARS-CoV-2 positive sample from the reference collection at VIDRL; all other specimens were known negatives. The positive samples varied in viral load, having previously 
tested at a range of cycle threshold $(\mathrm{Ct})$ values ranging from 19.3 to 35.6 in our standard diagnostic RNA-dependent RNA polymerase (RdRp) gene rRTPCR assay. ${ }^{5}$ Eight pools of each size were designed for use in the study.

\section{Diagnostic testing of clinical samples}

A total of 10,312 and 19,388 clinical samples were pooled for testing in March and May 2020, respectively. Samples from lower acuity settings (e.g., outpatient clinics) were selected for pooling, whereas samples from hospital inpatients, healthcare workers, and outbreak investigations-representing more urgent and/or higher prevalence settings - were specifically excluded from pooling. A large variety of swabs was received, including dry swabs to which viral transport medium was added during initial specimen processing.

\section{Sample pooling}

An equal amount of fluid from each specimen in a pool was combined into a single tube to give a final volume of $800 \mu \mathrm{L}$ (i.e., for a four-sample pool, 200 $\mu \mathrm{L}$ was aliquoted from each sample). The mixture was then vortexed for 5 seconds prior to nucleic acid extraction. Individual samples were retained for further testing should the pool test positive.

\section{Nucleic acid extraction and complementary DNA (cDNA) synthesis}

For viral RNA, $200 \mu \mathrm{L}$ of pooled sample was extracted with the QIAamp 96 Virus QIAcube HT kit (Qiagen, Germany) on the QIAcube HT System (Qiagen) according to manufacturer's instructions. Purified nucleic acid was then immediately converted to cDNA by reverse transcription with random hexamers using the SensiFAST cDNA Synthesis Kit (Bioline Reagents, UK) according to manufacturer's instructions. cDNA was used immediately in the rRT-PCR or stored at $-20^{\circ} \mathrm{C}$.

\section{SARS-CoV-2 rRT-PCR}

Three microlitres of cDNA was added to a commercial real-time PCR master mix (PrecisionFast qPCR Master Mix; Primer Design, UK) in a $20 \mu \mathrm{L}$ reaction mix containing primers and probe with a final concentration of $0.9 \mu \mathrm{M}$ and $0.2 \mu \mathrm{M}$ for each primer and the probe, respectively.

Primary screening of pooled samples was performed with a SARS-CoV-2 rRT-PCR targeting the RdRp gene..$^{5}$ RdRp-positive pools were then 'deconstructed', with each individual sample within that positive pool undergoing nucleic acid extraction and testing with rRT-PCRs targeting the E and $\mathrm{N}$ genes for confirmation. ${ }^{1}$ SARS-CoV-2 was reported as 'detected' in an individual sample if either the $\mathrm{E}$ or $\mathrm{N}$ gene was detected, as the RdRp gene detection in the pool could then be attributed to that sample, thereby fulfilling the Australian Public Health Laboratory Network recommendation of two targets for the detection of SARS-CoV-2.

An in-house positive extraction control, a negative control and a positive control were included with each PCR run. Thermal cycling and rRT-PCR analyses for all assays were performed on the ABI 7500 FAST real-time PCR system (Applied Biosystems, USA) with the following thermal cycling profile: $95^{\circ} \mathrm{C}$ for $2 \mathrm{~min}$, followed by $45 \mathrm{PCR}$ cycles of $95^{\circ} \mathrm{C}$ for $5 \mathrm{~s}$ and $60^{\circ} \mathrm{C}$ for $25 \mathrm{~s}$

\section{Statistical analysis}

Assay sensitivity was compared for each pool size used in the preimplementation study. The delta $\mathrm{Ct}$ value $(\Delta \mathrm{Ct})$ was defined as the absolute increase in $\mathrm{Ct}$ value when the pooled sample was tested compared to when the positive sample was tested individually. Therefore, a positive $\Delta \mathrm{Ct}$ value (i.e., an increase in $\mathrm{Ct}$ value in the pooled sample) represents the loss of PCR sensitivity attributable to sample pooling. The expected $\Delta \mathrm{Ct}$ value for each pool size was calculated based on the predicted dilution of the positive sample in the pool. Statistical significance of the mean $\Delta \mathrm{Ct}$ values was assessed using the paired sample t-test.

The interaction between the prevalence of SARS-CoV-2 in the test population and the efficiency of the various pool sizes was examined for a disease prevalence ranging from $0.2 \%$ to $20 \%$. As prevalence rises, the probability of positive pools requiring deconstruction and further individual testing rises with it. The expected number of tests required per 1000 individuals for pooled testing was calculated based on the method described by Black et al. ${ }^{7}$ using the open source R Shiny app (www.chrisbilder.com/shiny). This method requires calculation of the probability of a pooled sample testing positive, and this probability is derived from assay sensitivity and specificity as well as the prevalence of disease. For the purposes of this study, the sensitivity and specificity of the test were assumed to be fixed at $99 \%$.

\section{RESULTS}

\section{Sensitivity of pool sizes}

The mean $\mathrm{Ct}$ of the positive sample in each pool, the mean $\mathrm{Ct}$ of the pooled sample, and the mean $\Delta \mathrm{Ct}$ across the different pool sizes are shown in Table 1. All positive samples in each pool were successfully identified by retesting of individual samples as described in the study protocol.

\section{Pooling logistics}

The approximate time taken for pool assembly was $30 \mathrm{~min}$ for two-sample pools, $60 \mathrm{~min}$ for four-sample pools, and 120 min for eight-sample pools in a standard 96-well format. Oversight of the pool assembly process was provided by a second staff member to mitigate potential laboratory error. In addition, careful tracking of positive pools and accompanying worksheets was required to maintain traceability of results throughout the PCR workflow. After trialling pools of two, four, or eight samples, larger pools were not trialled, as eight-sample pools were considered the upper limit of what was feasible in our laboratory. This was based on the observed widening in $\Delta \mathrm{Ct}$ as pool sizes increased from two to eight samples; increasing logistical complexity and time required for pool assembly and deconstruction; together with increasing likelihood that positive pools will necessitate deconstruction and testing of individual samples, abrogating the efficiency benefit.

\section{Interaction of SARS-CoV-2 prevalence, pool size and efficiency}

Efficiency of pooling, expressed as the expected number of PCR reactions required for the testing of 1000 individual patients - inclusive of initial pool testing and deconstruction of positive pools - varied with disease prevalence and pool size (Table 2, Fig. 1). The maximum proportion of reagent savings for two, four, and eight sample pooling is $50 \%, 75 \%$, and $87.5 \%$ respectively, hence there are diminishing marginal gains in savings as pool size increases.

Table 1 Mean $\Delta \mathrm{Ct}$ for each pool size, representing the loss of PCR sensitivity attributable to pooling

\begin{tabular}{|c|c|c|c|c|c|}
\hline $\begin{array}{l}\text { Pool size } \\
\text { ( } n=8 \text { pools per pool size) }\end{array}$ & $\begin{array}{l}\text { Mean Ct of positive } \\
\text { sample (range) }\end{array}$ & $\begin{array}{l}\text { Mean Ct of pooled } \\
\text { sample (range) }\end{array}$ & Expected $\Delta \mathrm{Ct}$ & $\begin{array}{l}\text { Mean } \Delta \mathrm{Ct} \\
\text { (range) }\end{array}$ & $p$ value \\
\hline Four samples & $30.4(19.3-35.6)$ & $32.7(21.7-37.2)$ & 2.0 & $2.3(0.6-3.1)$ & $<0.0001$ \\
\hline Eight samples & $28.8(23.0-35.0)$ & $31.8(26.0-38.9)$ & 3.0 & $3.0(2.3-3.9)$ & $<0.0001$ \\
\hline
\end{tabular}


Table 2 Expected number of PCR reactions required for testing of 1000 samples according to pool size and disease prevalence (inclusive of initial pool testing and deconstruction of positive pools)

\begin{tabular}{|c|c|c|c|c|c|c|}
\hline \multirow[t]{2}{*}{ Prevalence of COVID-19 (\%) } & \multicolumn{2}{|c|}{ Two-sample pools } & \multicolumn{2}{|c|}{ Four-sample pools } & \multicolumn{2}{|c|}{ Eight-sample pools } \\
\hline & PCR reactions & Reagent savings & PCR reactions & Reagent savings & PCR reactions & Reagent savings \\
\hline 0.2 & 524 & $48 \%$ & 278 & $73 \%$ & 160 & $84 \%$ \\
\hline 0.5 & 530 & $48 \%$ & 289 & $72 \%$ & 183 & $82 \%$ \\
\hline 1 & 539 & $47 \%$ & 308 & $70 \%$ & 220 & $78 \%$ \\
\hline 2 & 558 & $46 \%$ & 345 & $67 \%$ & 290 & $72 \%$ \\
\hline 5 & 614 & $42 \%$ & 450 & $58 \%$ & 471 & $56 \%$ \\
\hline 10 & 706 & $36 \%$ & 606 & $45 \%$ & 701 & $37 \%$ \\
\hline 15 & 789 & $32 \%$ & 734 & $37 \%$ & 851 & $26 \%$ \\
\hline 20 & 871 & $28 \%$ & 845 & $30 \%$ & 954 & $21 \%$ \\
\hline
\end{tabular}

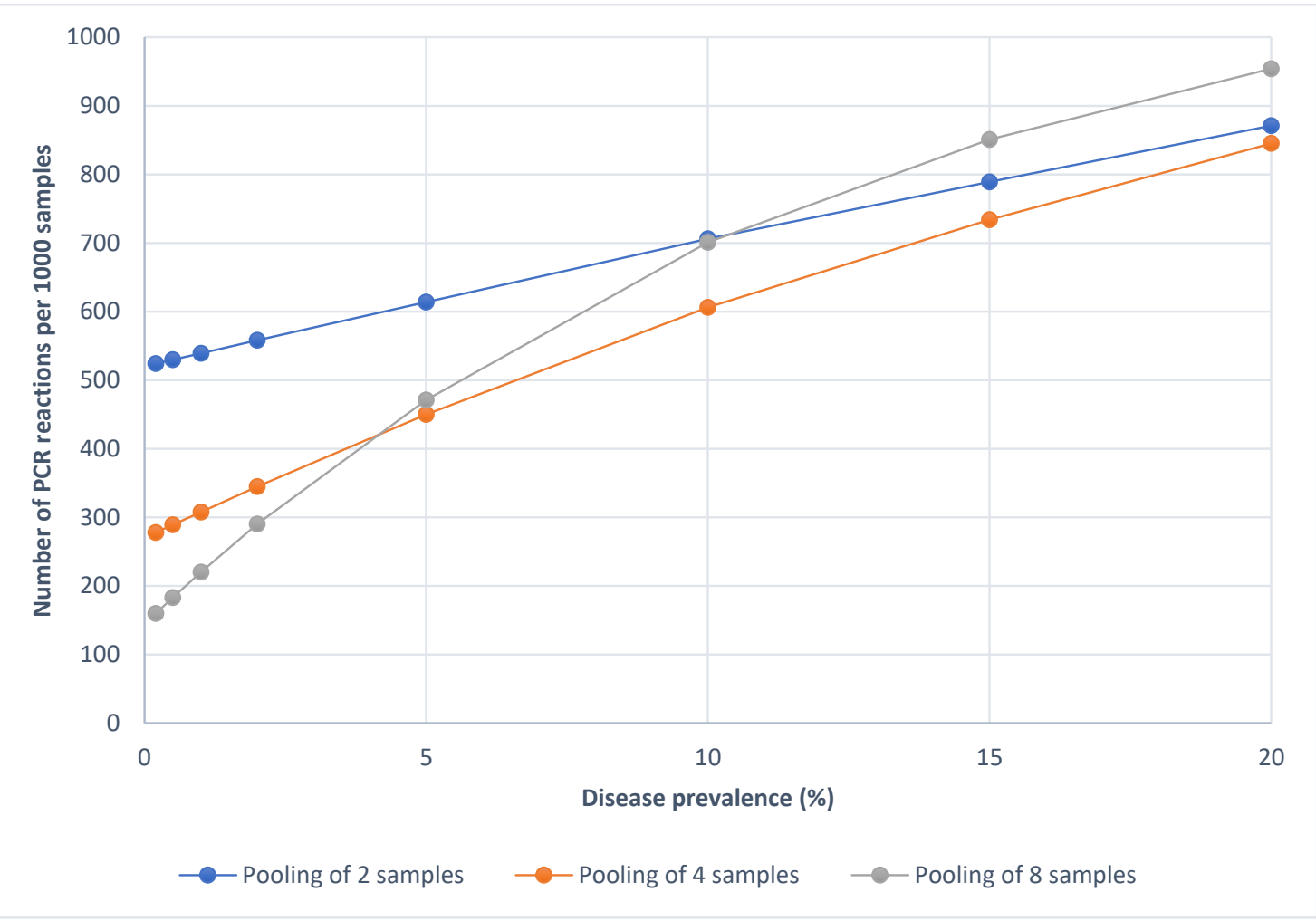

Fig. 1 Expected number of PCR reactions required per 1000 samples plotted against disease prevalence.

\section{Testing of clinical samples}

In March 2020, when the COVID-19 pandemic was in its early stages, and VIDRL was performing diagnostic testing for all of Victoria, testing demand rose to more than 3000 samples daily. As disease prevalence was $<0.5 \%$ at the time, pooled testing was performed using eight-sample pools. After a short period of peak demand was managed, our laboratory scaled back to four-sample pools which we considered to offer the best balance of efficiency and sensitivity. The peak daily throughput achieved in this period was 2222 results reported, compared to a normal daily throughput of approximately 100 respiratory virus RT-PCR tests. Four-sample pooling was again implemented in May 2020, during a high-throughput testing 'blitz' targeting low-risk individuals in the community with the goal of detecting asymptomatic cases. A total of 19,388 samples were tested over a 16-day period; 16,609 within 7 days, and with a peak day seeing 3341 results reported. Overall results of these two testing periods are summarised in Table 3.

\section{Risk mitigation}

Two significant laboratory errors occurred during the first period of high throughput testing using this pooling strategy, and these informed protocol modifications. In each case, error detection and rectification before the next working day obviated adverse clinical impact. Both were human errors; one involving inaccurate manipulation of a sample, and the other being incorrect orientation of a 96-well sample block. The extremely high test throughput and the relative novelty of the pooling protocol were likely cofactors. With pooling, the potential impact of error is magnified by the pooled sample size. Part of our risk mitigation in response to this error was 
Table 3 Summary of results for pooled testing of clinical samples during two periods of high demand

\begin{tabular}{|c|c|c|c|}
\hline Description & $\begin{array}{l}\text { 8-sample pooling } \\
\text { (March 2020) }\end{array}$ & $\begin{array}{l}\text { 4-sample pooling } \\
\text { (March 2020) }\end{array}$ & $\begin{array}{l}\text { 4-sample pooling } \\
\text { (May 2020) }\end{array}$ \\
\hline RdRp-positive pools & 8 & 49 & 3 \\
\hline Pools with one positive sample & 8 & 41 & 3 \\
\hline Pools with two positive samples & 0 & 8 & 0 \\
\hline Overall positivity rate & $0.4 \%$ & $0.6 \%$ & $0.02 \%$ \\
\hline
\end{tabular}

holding back reporting of negative pooled samples on each 96-well block until individual testing and analysis of positive pools was complete.

\section{Inter-laboratory implementation of pooling protocol}

Four-sample pooling was successfully implemented by the Microbiological Diagnostic Unit Public Health Laboratory, Doherty Institute (MDU PHL) using the Standard Operating Procedure (SOP) provided by VIDRL (refer to Appendix A for a sample workflow). MDU PHL integrated the pooling process as described, but otherwise retained their in-house testing method-including RNA extraction, PCR process, and gene target-which was different to the method described in this paper. During the 'blitz' in May 2020, 795 pools representing 3180 clinical samples were tested. Only one pooled sample tested positive, and the single positive sample in that pool was successfully identified on further testing.

\section{DISCUSSION}

In this study, we demonstrate that pooling is a viable diagnostic strategy for SARS CoV-2 detection in clinical samples, with which relative sparing of nucleic acid extraction kits and PCR reagents could be achieved and test throughput could be significantly increased. Pools of two, four and eight samples could be processed with modified RT-PCR workflows in the context of a very busy diagnostic laboratory setting.

As expected, test sensitivity diminished and testing efficiency increased in proportion to pool size. The mean $\Delta \mathrm{Ct}$ values obtained were in keeping with previous reports ${ }^{8,9}$ and close to the expected $\Delta \mathrm{Ct}$ for each pool size. Two-sample pools were found to impart only a small $\Delta \mathrm{Ct}$ of 0.6 , but produced insufficient efficiency gains to justify the workflow change to pooling. Pools of eight produced the greatest efficiency gains at low disease prevalences $(0-5 \%)$ likely to be seen in the early phases of an outbreak, but the significant mean $\Delta \mathrm{Ct}$ of 3.0 suggests that this strategy should be reserved for populations with extremely low pre-test probabilities, such as the asymptomatic general population, and only when testing is required at a scale too high to be achievable by other means. The additional workload of individually testing samples from positive pools of this size becomes impractical in high-prevalence settings. Pools of four appear to provide an efficiency gain over the whole range of prevalences of up to $20 \%$, however the gains diminish above $10 \%$ prevalence. It would appear that four-sample pools may be considered even when reagents are plentiful, as part of a dedicated workflow for testing large numbers of samples with very low pre-test probability, provided these can be reliably separated from samples requiring tests with maximum sensitivity due to patient disease severity, vulnerability, or the public health risk they potentially represent.

Pool assembly added a complex step to the test workflow, and required focus and precision from staff. To mitigate the potential for laboratory error, we found it was best managed in teams of two experienced staff: one to perform the aliquoting of samples, and the other to provide oversight and assemble the individual pools in separate racks. Even so, we experienced human errors during initial implementation, necessitating a change in our reporting protocol as discussed above. Pooling will typically be implemented in the context of high test demand, increasing the risk of error. Consequence of error also potentially increases due to involvement of multiple samples in each pool. Hence risk mitigation is an important part of pooling implementation planning.

Pooling significantly increases the total test throughput achievable in a working day — up to 3341 in our study — and hence reduces mean turnaround times. However, turnaround time via a pooled workflow is inherently slower than an individual sample testing workflow due to additional steps of pool assembly and subsequent individual testing of samples in positive pools. Urgent, high priority samples may be best managed separately from the pooled workflow if this can be achieved. It was also evident that as pooling increases laboratory throughput, pre-analytical stages in specimen reception and data entry are likely to become rate limiting unless resources are available to proportionally upscale these processes.

The laboratory governance aspects of pooling have to be taken into consideration, and these vary by region. For example, the United States Food and Drug Administration has issued Emergency Use Authorisations for SARS-CoV-2 in vitro diagnostic devices where pooling is included in these approvals. $^{10}$ The Australian Therapeutic Goods Administration has not provided specific exemptions for pooling. Hence, modifications to the workflow of commercial SARS-CoV-2 PCR kits may lead to a reclassification of these kits as 'in-house in vitro diagnostic devices', which requires that each laboratory validate them to standards set by the National Pathology Accreditation Advisory Council. ${ }^{11}$ Laboratories should account for local policies and regulations during the planning phase for sample pooling.

This study has several weaknesses. Firstly, it is not possible to design a single generic pooling workflow that is applicable to all laboratories. However, the workflow developed in this study has been successfully implemented in at least one other laboratory (MDU PHL) which used a completely different testing method. This demonstrates the transferability of our workflow. Secondly, the clinical impact of the slight reduction in assay sensitivity imparted by pooling was not formally assessed in this study. However, samples with low pre-test probability were specifically selected for pooling, and it is 
not possible to fully evaluate the false-negativity rate of pooling without large scale parallel testing of individual samples. The expected loss of clinical sensitivity will vary depending on the pre-pooling $\mathrm{Ct}$ distribution, which itself is dependent on the PCR assay used and test population. Furthermore, what an acceptable loss of sensitivity might be will differ according to the epidemiological scenario. Further studies are required to examine these outcomes.

\section{CONCLUSIONS}

In this study, we demonstrate that pooling is a viable strategy for SARS-CoV-2 testing in low-prevalence settings, provide a sample workflow, and report on the successful implementation of this workflow across two laboratories during periods of high testing demand.

Acknowledgements: The authors would like to acknowledge all staff at VIDRL and MDU PHL for their work throughout the SARS-CoV-2 pandemic.

Conflicts of interest and sources of funding: VIDRL's funding for public health laboratory testing for Victoria is provided by the Victorian Department of Health and Human Services. The authors state that there are no conflicts of interest to disclose.

\section{APPENDIX A. SUPPLEMENTARY DATA}

Supplementary data to this article can be found online at https://doi.org/10.1016/j.pathol.2020.09.005.

Address for correspondence: Dr Brian S. W. Chong, Victorian Infectious Diseases Reference Laboratory, The Peter Doherty Institute for Infection and Immunity, 792 Elizabeth St, Melbourne, Vic, 3000, Australia. E-mail: bcswen86@gmail.com

\section{References}

1. Corman VM, Landt O, Kaiser M, et al. Detection of 2019 novel coronavirus (2019-nCoV) by real-time RT-PCR. Euro Surveill 2020; 25: 2000045.

2. Morandi PA, Schockmel GA, Yerly S, et al. Detection of human immunodeficiency virus type 1 (HIV-1) RNA in pools of sera negative for antibodies to HIV-1 and HIV-2. J Clin Microbiol 1998; 36: $1534-8$.

3. Mine H, Emura H, Miyamoto M, et al. High throughput screening of 16 million serologically negative blood donors for hepatitis B virus, hepatitis $\mathrm{C}$ virus and human immunodeficiency virus type- 1 by nucleic acid amplification testing with specific and sensitive multiplex reagent in Japan. J Virol Methods 2003; 112: 145-51.

4. Hogan CA. Sahoo MK, Pinsky BA. Sample pooling as a strategy to detect community transmission of SARS-CoV-2. JAMA 2020; 323: 1967-9.

5. Caly L, Druce J, Roberts J, et al. Isolation and rapid sharing of the 2019 novel coronavirus (SARS-CoV-2) from the first patient diagnosed with COVID-19 in Australia. Med J Aust 2020; 212: 459-62.

6. Australian Public Health Laboratory Network. Public Health Laboratory Network (PHLN) guidance on laboratory testing for SARS-CoV-2 (the virus that causes COVID-19). Updated 13 Aug 2020; cited 25 Aug 2020. https://www.health.gov.au/resources/publications/phln-guidanceon-laboratory-testing-for-sars-cov-2-the-virus-that-causes-covid-19.

7. Black MS, Bilder CR, Tebbs JM. Optimal retesting configurations for hierarchical group testing. J R Stat Soc Ser C Appl Stat 2015; 64: 693-710.

8. Sahajpal NS, Mondal AK, Njau A, et al. Proposal of RT-PCR-based mass population screening for severe acute respiratory syndrome coronavirus 2 (coronavirus disease 2019). J Mol Diagn 2020; Jul 30: https:// doi.org/10.1016/j.jmoldx.2020.07.001.

9. Lohse S, Pfuhl T, Berko-Gottel B, et al. Pooling of samples for testing for SARS-CoV-2 in asymptomatic people. Lancet Infect Dis 2020; Apr 28: https://doi.org/10.1016/S1473-3099(20)30362-5.

10. United States Food and Drug Administration. Pooled sample testing and screening testing for COVID-19. Cited 25 Aug 2020. https://www.fda. gov/medical-devices/coronavirus-covid-19-and-medical-devices/pooledsample-testing-and-screening-testing-covid-19.

11. National Pathology Accreditation Advisory Council. Requirements for the development and use of in-house in vitro diagnostic medical devices (IVDs). 27 Jun 2018; cited 25 Aug 2020. https://www1.health.gov.au/ internet/main/publishing.nsf/Content/health-npaac-dhaivd-2018. 